\title{
Specific autonomy recovery programme in a comprehensive rehabilitation on functionality and respiratory parameters in oncological patients with dyspnoea. Study protocol
}

Eduardo José Fernández-Rodríguez ${ }^{1,2,3}$, Jesús González-Sánchez ${ }^{1,3}$, Ana Silvia Puente-González,3, José Ignacio Recio-Rodríguez ${ }^{1,3}$, Celia Sánchez-Gómez ${ }^{3,4}$, Roberto Méndez-Sánchez ${ }^{1, *^{*}}$ (i), Juan Jesús Cruz-Hernández ${ }^{2,3,5}$ and María Isabel Rihuete-Galve ${ }^{1,2,3}$

\begin{abstract}
Background: Survival in cancer patients has increased exponentially in recent years, with multiple side effects caused by treatments. Cancer-related asthenia and dyspnea are among them, which represent a serious health problem, with considerable limitations and reduced quality of life. An implementation of the conventional clinical practice, developed through physical exercise, may be useful in controlling dyspnoea. This study aims to compare the effects of a comprehensive rehabilitation implementing a programme of multimodal physical exercise with a specific autonomy recovery programme, versus an isolated intervention using the physical exercise programme alone, on the functionality, physical performance and respiratory parameters in oncologycal patients with dyspnea.

Methods: This is a protocol por an experimental, prospective, randomized, parallel-controlled clinical trial, with two arms design of fixed assignment with an experimental and control groups. It will conduct in the Oncology Hospitalisation Unit at the University Hospital Complex of Salamanca, using consecutive sampling to select 50 participants with oncological dyspnoea who are hospitalised at the time of inclusion. After baseline assessment, participants will be randomised into the groups. Experimental group will complete Comprehensive Rehabilitation with the autonomy recovery and the multimodal exercise programmes, and in the control group, only the multimodal exercise programme will be carried out. The primary outcomes will be basic activities of daily living (Barthel Index) and degree of dyspnoea (MRC scale). Additionally, physical performance will be evaluated with the Short Physical Performance Battery (SPPB), as will the oxygen saturation in the blood using pulse oximetry, fear/ avoidance of movement with the Tampa Scale of Kinesiophobia (TSK), and the quality of life of the oncology patient (ECOG performance scale).
\end{abstract}

\footnotetext{
*Correspondence: ro_mendez@usal.es

'Department of Nursing and Physiotherapy, University of Salamanca,

Salamanca, Spain

${ }^{3}$ Institute of Biomedical Research of Salamanca (IBSAL), Salamanca, Spain

Full list of author information is available at the end of the article
}

C C The Author(s). 2021 Open Access This article is licensed under a Creative Commons Attribution 4.0 International License, which permits use, sharing, adaptation, distribution and reproduction in any medium or format, as long as you give appropriate credit to the original author(s) and the source, provide a link to the Creative Commons licence, and indicate if changes were made. The images or other third party material in this article are included in the article's Creative Commons licence, unless indicated otherwise in a credit line to the material. If material is not included in the article's Creative Commons licence and your intended use is not permitted by statutory regulation or exceeds the permitted use, you will need to obtain permission directly from the copyright holder. To view a copy of this licence, visit http://creativecommons.org/licenses/by/4.0/ The Creative Commons Public Domain Dedication waiver (http://creativecommons.org/publicdomain/zero/1.0/) applies to the data made available in this article, unless otherwise stated in a credit line to the data. 
Discussion: The results of this study may be translated to clinical practice, incorporating a specific autonomy recovery programme into comprehensive rehabilitation programmes of care for cancer patients with dyspnoea. Increase in the survival of patients with cancer includes multiple side effects as cancer-related asthenia and dyspnea, which represents a serious health problem. The current study addresses to improve the conventional clinical practice by proposing an integral, rehabilitative approach, to implement education and training for oncology patients with dyspnea to increase their quality of life.

Trial registration: ClinicalTrials.gov; ID: NCT04766593. (February 23, 2021).

Keywords: Cancer, Oncology, Dyspnoea, Nursing, Comprehensive rehabilitation, Autonomy, Exercise, Functionality, Quality of live

\section{Background}

In recent years, the improvement in cancer treatments and broad support for preventive strategies have enabled us to reach a higher rate of early diagnosis and a better understanding of the oncologic pathology itself, thus achieving an exponential increase in the five-year survival of patients with cancer [1].

Linked to that increase in survival, and due to the resulting increase in the lines of treatment employed, we are seeing greater side effects, which have repercussions in aspects such as the patients' functionality and their quality of life [2]. Some of these side effects include cancer-related asthenia, anxiety, and dyspnoea [3]. Dyspnoea may represent a serious health problem, with considerable limitations for the individuals. In some patients with advanced-stage cancer, this dyspnoea may represent a clinical sign of the final phase of the disease [4]. Approximately $41 \%$ of the patients in palliative care have dyspnoea, and $46 \%$ of those describe it as being moderate or severe $[5,6]$.

The majority of patients perceive this dyspnoea to be a limiting factor that is out of their control, which leads them to adopt behaviours which have negative repercussions on their functionality [7], such as cancer patients creating patterns of fear/avoidance of movement or of physical activity, as is often the case also with patients with chronic pain, chronic fatigue syndrome, or fibromyalgia $[8,9]$. It is similar to when patients with respiratory problems decrease their level of activity to adapt to their symptomatology. This favours a worsening of their physical state and of the dyspnoea upon exertion, known as the "cycle of the respiratory patient" [10].

To control the dyspnoea, we believe that the measures employed in conventional clinical practice, which have been mainly developed through physical exercise, may be implemented. Reeducation and work on the activities of daily living, together with a programme of physical exercise, as part of an integral approach of functional reeducation, may be a good intervention for dyspnoea in oncology patients
[11]. For this reason, in this clinical trial we are proposing an intervention with an integral perspective that is based on a rehabilitative approach, with the objective of integrating education and training of the oncology patient with dyspnoea in the adaptation of their day-to-day activities while their cardiopulmonary reserves are gravely reduced, with the aim of recovering their capacity to carry out their occupational roles.

After analysing previous studies, we have observed that the most-employed intervention is physical exercise, which has been shown to give good results in the majority of dyspnoea cases [12, 13]. Additionally, it appears that multimodal physical exercise programmes, which combine different types of exercise, such as aerobic exercise and strength training primarily, appear to have better overall results in cancer patients [14-16]. However, there are not many studies which correlate whether this improvement in symptoms has had positive repercussions on the patients' functionality when carrying out their activities of daily living. We believe that, in addition to employing physical exercise to improve the patients symptomatology, this intervention should be geared towards increasing the individuals autonomy, and that this will make it possible to generalise in their daily life the symptomatic clinical effects obtained through the therapeutic process, which will be inexorably linked to an improvement in their quality of life.

As such, we are proposing as an intervention an Oncological Functional Reeducation Programme, to be used in addition to the intervention with physical exercise. It would be a reeducation in the completion of the activities of daily living, so as to foment the recovery of personal autonomy, and thus to observe to what degree this improves the results in clinical practice. The findings will provide important insight into the development of an effective care model with a comprehensive rehabilitation programme that includes a specific autonomy recovery programme in oncological patients with dyspnoea. 


\section{Methods/design}

\section{Aims}

In accordance with that laid out above, the primary aim of this study is to contrast our hypothesis, that the intervention using a specific programme for the recovery of autonomy by means of reeducation on the activities of daily life, when added to multimodal physical exercise, will increase the beneficial effects of physical exercise as an isolated intervention on the functionality, physical performance, and respiratory parameters in oncology patients with dyspnea. The objectives seek to compare the effects produced by the implementation of a programme of multimodal physical exercise and a specific programme for recovering autonomy, with an isolated intervention using physical exercise, on the functionality and physical performance of cancer patients with dyspnoea.

\section{Design}

This study is an experimental, prospective, randomized, parallel-controlled clinical trial, with two arms design of fixed assignment with an experimental and a control groups. It will conduct during a year in the Oncology Hospitalisation Unit at the University Hospital Complex of Salamanca (CAUSA), using consecutive sampling to select the participants with oncological dyspnoea who are hospitalised at the time of inclusion.

\section{Sample/participants \\ Participants}

The patients will be recruited from the Oncology Hospitalisation Unit at the CAUSA, and must meet the following selection criteria: A) Inclusion criteria: Participants must have a pathological diagnosis of an oncological disease, be over 18 years of age, be hospitalised at the time of recruitment in the Oncology Unit at CAUSA, meet dyspnoea parameters of 2 or more points on the Medical Research Council scale (MRC), receive fewer than 85 points on the Barthel Index, and have signed an informed consent form indicating voluntary agreement to participate in the study. B) Exclusion criteria: not having an adequate cognitive state to be able to comprehend and follow the orders provided (fewer than 23 points on the Mini Mental State Examination, MMSE), having a haemoglobin level lower than $10 \mathrm{~g} / \mathrm{dL}$, being an active smoker at the time of recruitment. C) Withdrawal criteria: disease progression which brings the patient to a terminal situation or to death, and non-completion of the follow-up and final assessments.

\section{Sample size}

The sample size has been estimated based on the potential modification of the most stringent of the principal variables of the study-the score on the Barthel Index. For this, as a reference, we have used the results obtained in a study with similar characteristics, in which the scoring on the Barthel Index was modified by 7.5 points [17]. With these premises, accepting an alpha risk of 0.05 and a beta risk of 0.2 in a bilateral contrast, we will need 25 subjects in the first group and 25 subjects in the second group in order to detect a difference greater than or equal to 7.5 units. The assumed common standard deviation is 8.3 . We estimate a rate of $20 \%$ lost to follow-up. The sample size was estimated using the programme EPIDAT 4.2.

\section{Sample assignment}

After meeting the selection criteria listed above and being recruited for the study, the individuals will be assigned via a simple randomisation process to one of the two conditions of the study: Experimental Condition or Control Condition. As detailed in Fig. 1.

\section{Randomisation}

We will employ a simple randomisation process, which consists of generating a table of random numbers that is the same size as the estimated sample in the study. This will be done using Microsoft Excel 2020. Each of the randomised numbers will be assigned a condition: for even numbers, the subject will be assigned to the Experimental condition, and for odd numbers, they will be assigned to the Control condition. The work of the randomisation sequencing, the recruitment of participants, and of assigning them to a group will be completed by research personnel who are not related to the assessments nor to the implementation of the intervention, which will prevent potential biases in the study.

\section{Blinding}

Given the nature of the intervention, the participating subjects will not be blinded. However, to minimise any contamination between groups, the investigator responsible for the measurements of the study at each assessment and for the statistical analysis will be blinded, thus increasing the scientific quality in the study process.

\section{Procedures and data collection \\ Evaluations and study plan}

Three assessments will be completed in this study: one at the beginning, another after 7 days, and a final one before the patient is discharged from hospital. After recruitment and before randomisation and assignment to the corresponding group, all individuals will complete a baseline assessment (BA), which includes recording the independent variables and the variables of the primary and secondary results of the study. Then, while the patients are still in hospital, the subjects will be randomly assigned to the control or the experimental group, with the corresponding intervention applied in each of them. 


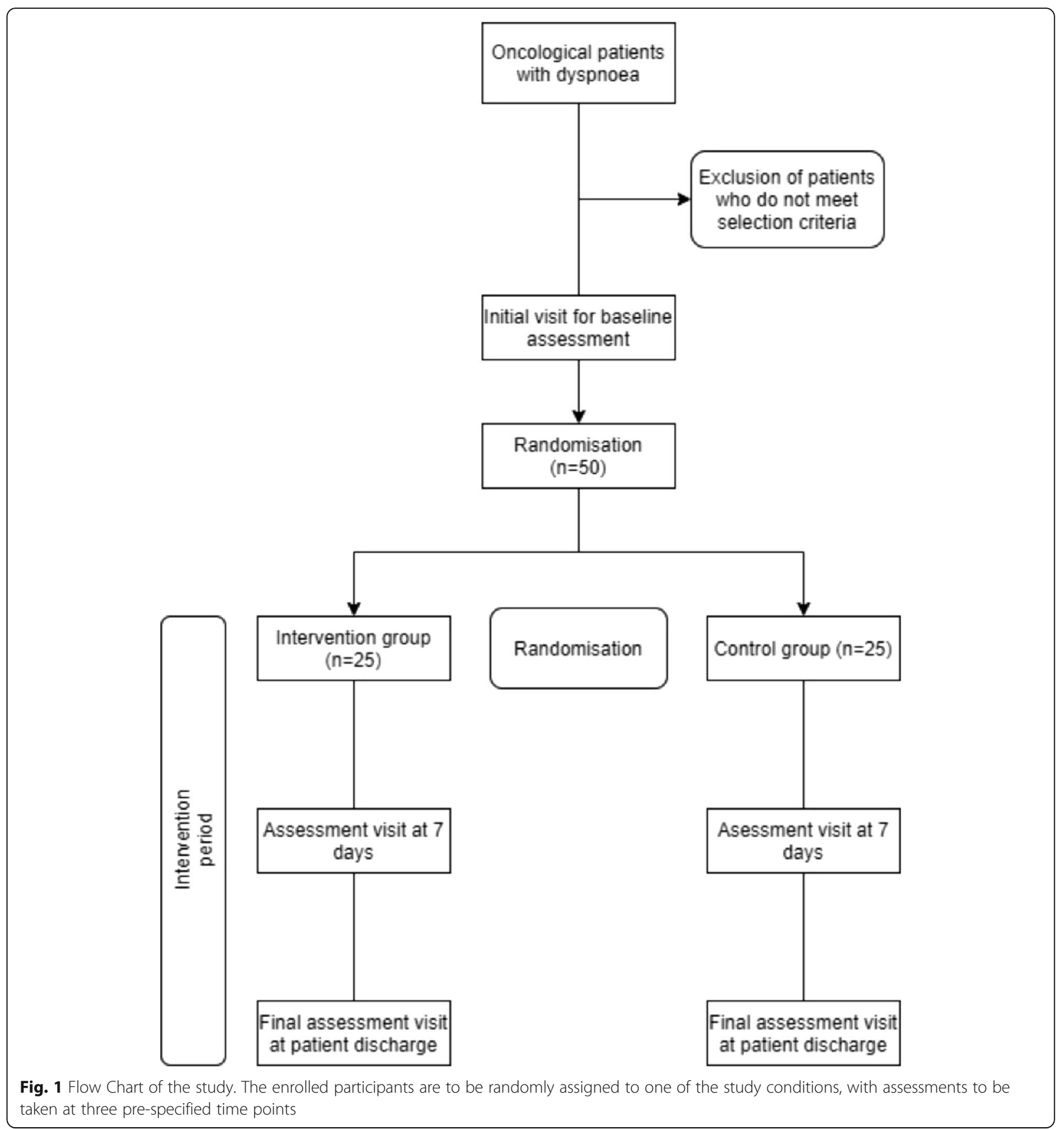

Later, two more assessments will be completed, one after 7 days, considered to be the follow-up assessment (FUA), and another at the time of discharge from the hospital, considered to be the final assessment (FA). This can be observed in more detail in Table 1 .

\section{Description of the variables}

For the principal variable being manipulated, we will study dyspnoea (Medical Research Council, MRC) and the degree of dependence in carrying out the activities of daily living (Barthel Index, BI). And as secondary variables, we have the patient's physical performance (via the Short Physical Performance Score, SPPB), their fear/avoidance of movement (Tampa Scale of Kinesiophobia-Fatigue, TSK-F), the oxygen saturation in the blood (pulse oximetry), and the quality of life of the oncology patient (ECOG performance status scale). Other intervening variables include those gathered from the patient's clinical 
Table 1 Timeline and dimensions assessed at the time points

\begin{tabular}{|c|c|c|c|c|c|c|c|c|}
\hline & \multicolumn{8}{|c|}{ STUDY PERIOD } \\
\hline & \multirow{2}{*}{$\begin{array}{l}\text { Enrolment } \\
\text { Month 1-2 }\end{array}$} & \multirow{2}{*}{$\begin{array}{l}\text { Allocation } \\
\text { Month 2-3 }\end{array}$} & \multicolumn{5}{|c|}{ Post-allocation } & \multirow{2}{*}{$\begin{array}{l}\text { Close-out } \\
\text { M9-M12 }\end{array}$} \\
\hline TIMEPOINT & & & M4 & M5 & M6 & M7 & M8 & \\
\hline ENROLMENT: & & & & & & & & \\
\hline Eligibility screening & $\mathrm{X}$ & & & & & & & \\
\hline $\begin{array}{r}\text { Database } \\
\text { preparation }\end{array}$ & $\mathrm{X}$ & & & & & & & \\
\hline Informed consent & & $\mathrm{X}$ & & & & & & \\
\hline Allocation & & $\mathrm{X}$ & & & & & & \\
\hline INTERVENTIONS: & & & & & & & & \\
\hline $\begin{array}{l}\text { [Experimental } \\
\text { Intervention] }\end{array}$ & & & & & & & & \\
\hline $\begin{array}{r}\text { [Control } \\
\text { Intervention] }\end{array}$ & & & & & & & & \\
\hline ASSESSMENTS: & & & & & & & & \\
\hline $\begin{array}{r}\text { [BASELINE } \\
\text { ASSESSMENT] }\end{array}$ & & $\mathrm{X}$ & & & & & & \\
\hline $\begin{array}{c}\text { [FOLLOW-UP } \\
\text { ASSESSMENT] }\end{array}$ & & & & & & & & $\mathrm{X}$ \\
\hline $\begin{array}{r}\text { [FINAL } \\
\text { ASSESSMENT] }\end{array}$ & & & & & & & & $\mathrm{X}$ \\
\hline $\begin{array}{r}\text { DISSEMINATION } \\
\text { OF RESULTS: }\end{array}$ & & & & & & & & $\mathrm{X}$ \\
\hline
\end{tabular}


history, such as pathological diagnosis, number of lines of treatment, and socio-demographic and anthropometric data (sex, age, weight, height, and BMI).

\section{Tools employed in the evaluation of the variables}

1. "Barthel Index, (BI)" [18]: to evaluate dependence. Measures physical disability with proven validity and reliability. It is easy to apply and to interpret. Useful for evaluating the patient's functional independence in the activities of daily living (ADL). Scored from 0 to 100 , it quantifies the individual's degree of dependence.

2. "Medical Research Council dyspnoea scale (MRC)" [19], to evaluate dyspnoea. The aim is to make it possible for the patients to quantitatively grade their own dyspnoea in a simple, visual manner. It establishes 5 degrees of dyspnoea when carrying out activities.

3. "Short Physical Performance Score (SPPB)" [20], to evaluate physical performance. The short physical performance battery, proven in our area for primary health care, is a test designed specifically to predict disabilities, and it has demonstrated the capacity to predict adverse events, dependence, institutionalisation, and mortality.

4. The "Tampa Scale of Kinesiophobia-Fatigue (TSKF)" [8, 9], was developed to evaluate the fear of movement related to fatigue/pain. It has been proven effective in oncology patients and those with chronic fatigue syndrome. We will use the model with 11 items (TSK-F-11).

5. "Pulse oximetry" [21]: a non-invasive technique which uses a pulse oximeter to measure the saturation of oxygen $\left(\mathrm{Sat}_{2}\right)$ in the haemoglobin in circulating blood, usually in arterial blood.

6. "ECOG performance status scale" [22], is a practical way to measure the quality of life of cancer patients, whose life expectancies may change within a period of months, weeks, or even days. It was designed by the Eastern Cooperative Oncology Group (ECOG) in the United States and validated by the World Health Organization (WHO). The primary function of this scale is to objectify the patient's quality of life or performance status.

The variables will be recorded on an individual sheet for each patient and stored afterwards in a database designed specifically for this study.

\section{Interventions}

The conventional standard of care will be maintained in both groups, as well as the lines of treatment prescribed by the Medical Oncology Service of the
CAUSA. Additionally, all subjects in both groups will receive an individualised prescription of multimodal physical exercise.

In the experimental group, we will also implement an intervention with a programme for recovering the individual's autonomy by means of reeducation in the activities of daily living.

The oncological functional reeducation programme will comprise the following actions:

1. Multimodal physical exercise programme: this therapeutic measure will be applied for all individuals, in both the experimental group and the control group. It will consist of two short sessions each day, one in the morning and another in the afternoon, of 15-20 min each. The dose and load of the programme, as well as any special considerations, will always be adapted to the functional capacities of each patient, in accordance with the assessments carried out. Additionally, we will always take into consideration the patient's symptoms at the time, as there may be individual changes in a very short period of time. The directive will be to maintain a multimodal exercise programme to complete different exercises, including aerobic exercises, balance exercises, and low-load resistance training exercises targeting specific muscle groups, in both the upper quadrant and the lower quadrant. The sessions will be done following the recommendations of the American College of Sports Medicine (ACSM) [23], with an initial warm-up (2-3 min), a main part (8-12 min), and a final cool-down and relaxation ( $5 \mathrm{~min}$ ) It will be permitted-and recommended-to do the exercises with the patient's specific oxygen therapy range, if they have one. The patient's oxygen saturation levels will also be measured before, during, and after completing the exercise, to ensure that there are no clinical alterations, which may contraindicate the therapeutic practice.

2. Reeducation in the activities of daily living: Intensity and simplification of the activities and trainings of energy-saving techniques (EST). An individual training will be completed on the rules for the simplification of activities, consisting of [24]:

- Exhaustive organisation of the workspaces where the activities are to be carried out

- Adaptation of the work plans and placement of the objects within the patient's reach

- Prioritising basic self-care activities while sitting

- Movement control: doing the movements in a slow, coordinated, and harmonious manner, avoiding impulsive and vigorous movements 
- Alternating heavy activities with light activities, establishing the required rest times between them

- Combining periods of balanced activity with periods of rest

An individualised assessment will also be completed where we will prescribe the support products which will facilitate the patient's mobilisation when completing their activities of daily living, such as a walker with an adaptation for oxygen therapy (a portable oxygen concentrator).

And finally, we will complete an exhaustive daily $\log$ of the activity that the patient does, which we will in turn use to modify the same activity, adapting it to the patient's clinical situation at all times. In this way, we will establish a proper pattern, which will lead the patient to improve their autonomy, as they will be using all of their capacities available at the time, without exceeding them or discounting them. This will be done using a diary which the patient will complete each day and which the investigator responsible for the intervention will supervise daily.

\section{Data analysis}

The statistical analysis will be carried out with the intention to treat. In the descriptive analysis, the population is described by presenting the data as means and standard deviations for the continuous variables and as frequency distributions for the qualitative variables. To evaluate the comparability in the baseline assessment between the two study groups, we will employ the Chisquared test for qualitative variables and the Student's T-test for the comparison of the means between the two groups, in addition to the Kolmogorov-Smirnov test to find the normality of the sample. In the inferential analysis, we will consider a confidence interval of $95 \%(p<$ 0.05 ), and will use Student's T-test for related pre and post samples and for independent samples at the end of the study, in addition to a multivariate analysis of variance (MANOVA) with repeated measures (3 levels) to assess interactions between categorical variables, and to determine significant differences between groups and within groups. Additionally, to assess the magnitude of the change in the variables, the effect size of the interventions will be calculated as Cohen's d (small (0.2), medium (0.5), and large (0.8)) and the partial eta squared ( $\mathrm{n} 2 \mathrm{p}$ ) (small (0.01), medium (0.06), and large $(0.14)$ ), where significant, as appropriate with the statistical test applied [25]. We will use Pearson's Correlation Coefficient to analyse the correlations between the various intervening variables and the parameters established in the different questionnaires and scales employed. A survival follow-up analysis will be completed using the
Kaplan-Meier non-parametric method to learn the time of the intervention until the patient's discharge from hospital, and the survival curves of each of the groups in the study will be compared using the non-parametric logrank test. Processing and analysing the data will be done using the statistics pack SPSS 25.0.

\section{Ethical considerations}

The study will be carried out thanks to the authorisation of a Clinical Research Ethics Committee of the Area of Health of Salamanca (ID: PI 202007547), having obtained the prior written informed consent of the study subjects and in conformance with the Helsinki Declaration. In it, participants will be informed of the objectives of the project, as well as the risks and benefits of the interventions to be carried out, explained by one of the main investigators. The confidentiality of the subjects included will be guaranteed at all times, in conformance with the laws regarding the protection of personal data and biomedical investigation present in the articles of Spanish Organic Law 3/2018 of 5 December on the protection of personal data and the guarantee of digital rights, and in Regulation (EU) 2016/679 of the European Parliament and of the Council of 27 April 2016 on data protection (GDPR), and within the conditions established by Law $14 / 2007$ on biomedical investigation.

Significant modifications to the protocol (such as changes in the tools of evaluation, modifications to the selection criteria or to the interventions) will be communicated immediately to the Ethics Committee.

And since this is a randomised clinical trial, it follows the CONSORT guidelines, and it was registered. TRIAL REGISTRATION: ClinicalTrials.gov NCT04766593.

\section{Rigour}

This protocol study also follows the evidence-based recommendations of The SPIRIT 2013 Statement for the minimum content of a clinical trial protocol. And the design of the study also follows the evidence-based, minimum set of recommendations of the CONSORT 2010 Statement for conduct parallel-group randomized controlled trials (RCT), enabling readers to understand a trial's design, conduct, analysis and interpretation, and to assess the validity of its results.

\section{Discussion}

If it is true that there has been an exponential increase in the studies related to the implementation of physical exercise in the intervention of patients with respiratory pathologies, there still remains a problem with the generalisation that those individuals achieve that symptomatic improvement in the completion of their activities of daily living. 
The present study will attempt to investigate the influence that rehabilitative measures that are oriented exclusively towards functional recovery and adaptation will have with respect to the measures of physical exercise employed with oncological patients with respiratory symptomatology.

In our daily clinical practice, we have observed firsthand the beneficial effects of physical exercise in the physical improvement of patients with dyspnoea. But we have also observed difficulties with the generalisation of this physical improvement in terms of occupational performance.

Based on the results obtained, we consider it indispensable to establish specific care protocols which improve not only the symptomatology, but also the individual in a more integral manner, which we believe will have positive repercussions on their quality of life and on that of the people around them. Dyspnoea is a symptom that is clearly incapacitating and which increases individuals' dependence, which is then linked to an exceeding personal cost for their caretakers. By improving patients' levels of dependence, we are contributing to the reduction of this problem for the caretakers.

\section{Limitations}

The study follows all of the recommendations of CONSORT, but due to the nature of the intervention itself, the participating subjects will not be blinded. However, to minimise any contamination between groups, the investigator responsible for the study measurements at each assessment and the statistical analysis thereof, will be blinded.

\section{Dissemination plan}

The investigator team is planning a rapid and broad diffusion of the results so as to guarantee the maximum visibility of this study. With this aim, the results of the study will be published in open-access, peer-reviewed scientific journals. We will have at least one publication of the primary results, and others planned with the secondary results. This will complement the presentation of the study results at important national and international scientific conferences and seminars. Similarly, we will do a diffusion on social media and other media.

\section{How potential changes in the study will be approached}

Significant modifications to the protocol (such as change in the tools of evaluation, modifications to the selection criteria or to the interventions) will be communicated immediately to the bioethics committee.

\section{Conclusions}

This manuscript presents the protocol of a study aimed at assessing the results of a model of care based on a comprehensive rehabilitation combining a usual multimodal physical exercise programme and a specific programme of recovery of autonomy through activities of daily living training, on functionality, respiratory parameters and quality of life in oncology patients with dyspnea.

\section{Abbreviations \\ MRC: Medical research council; SPPB: Short physical performance score; ECOG: Eastern Cooperative Oncology Group; ADL: Activities of daily living; MMSE: Mini-mental state examination; BI: Barthel index; TSK-F: Tampa Scale of Kinesiophobia-Fatigue; WHO: World Health Organization; ACSM: American College of Sports Medicine; EST: Energy-saving techniques; EU: European Union}

\section{Acknowledgements}

The investigative team would like to express their appreciation to all of the patients who will take part in the study for their participation, as well as to all of our professional colleagues who will contribute in some way to the optimal development of this study.

\section{Authors' contributions}

EJFR, MIRG, and JJCH are the principal investigators; they designed the study and are responsible for the trial design and study procedures. CSG is an occupational therapist and is responsible for recruitment and the patients' information. ASPG and RMS are responsible for the interventions and for the supervision of the study and the participants. JIRR and JGS are responsible for the statistical analysis. JIRR, EJFR, RMS, CSG, and MIRG are responsible for the preparation of publications. All authors read and approved the final manuscript.

\section{Funding}

The investigators do not declare any type of financing for carrying out the study.

\section{Availability of data and materials}

Upon completion of the study, the datasets generated and/or analysed during the current study will be available to those investigators who request it through FAlRsharing of the repositories of research data at https://www. re3data.org/.

\section{Declarations}

\section{Ethics approval and consent to participate}

The study was approved by the Clinical Research Ethics Committee of the Area of Health of Salamanca (ID: PI 202007547), having obtained the prior written informed consent of the study subjects and in conformance with the Helsinki Declaration.

\section{Consent for publication}

Not applicable.

\section{Competing interests}

The authors declare that they have no competing interests.

\author{
Author details \\ ${ }^{1}$ Department of Nursing and Physiotherapy, University of Salamanca, \\ Salamanca, Spain. ${ }^{2}$ Medical Oncology Service, University Hospital Complex of \\ Salamanca, Salamanca, Spain. ${ }^{3}$ Institute of Biomedical Research of Salamanca \\ (IBSAL), Salamanca, Spain. ${ }^{4}$ Department of Developmental and Educational \\ Psychology, University of Salamanca, Salamanca, Spain. ${ }^{5}$ Department of \\ Medicine, University of Salamanca, Salamanca, Spain.
}

Received: 28 May 2021 Accepted: 9 June 2021

Published online: 05 July 2021

\section{References}

1. Bray F, Ferlay J, Soerjomataram I, Siegel RL, Torre LA, Jemal A. Global cancer statistics 2018: GLOBOCAN estimates of incidence and mortality worldwide 
for 36 cancers in 185 countries. CA Cancer J Clin. 2018;68(6):394-424. https://doi.org/10.3322/caac.21492.

2. Siegel RL, Miller KD, Jemal A. Cancer statistics, 2020. CA Cancer J Clin. 2020; 70(1):7-30. https://doi.org/10.3322/caac.21590.

3. Koelwyn GJ, Jones LW, Hornsby W, Eves ND. Exercise therapy in the management of dyspnea in patients with cancer. Curr Opin Support Palliat Care. 2012;6(2):129-37. https://doi.org/10.1097/SPC.0b013e32835391dc.

4. Nakano J, Hashizume K, Fukushima T, Ueno K, Matsuura E, Ikio Y, et al. Effects of aerobic and resistance exercises on physical symptoms in Cancer patients: a meta-analysis. Integr Cancer Ther. 2018;17(4):1048-58. https://doi. org/10.1177/1534735418807555.

5. Ripamonti C. Management of dyspnea in advanced cancer patients. Support Care Cancer. 1999;7(4):233-43. https://doi.org/10.1007/s005200050255.

6. Cooper J. Occupational therapy in oncology and palliative care. London: Wiley; 2006.

7. Migliore A. Improving dyspnea management in three adults with chronic obstructive pulmonary disease. Am J Occup Ther. 2004;58(6):639-46. https:// doi.org/10.5014/ajot.58.6.639.

8. Velthuis MJ, Van den Bussche E, May AM, Gijsen BC, Nijs S, Vlaeyen JW. Fear of movement in cancer survivors: validation of the modified Tampa scale of kinesiophobia-fatigue. Psychooncology. 2012;21(7):762-70. https://doi.org/1 0.1002/pon.1971.

9. Nijs J, Roussel N, Van Oosterwijck J, De Kooning M, Ickmans K, Struyf F, et al. Fear of movement and avoidance behaviour toward physical activity in chronic-fatigue syndrome and fibromyalgia: state of the art and implications for clinical practice. Clin Rheumatol. 2013;32(8):1121-9. https://doi.org/10.1 007/s10067-013-2277-4.

10. Silver JK, Gilchrist LS. Cancer rehabilitation with a focus on evidence-based outpatient physical and occupational therapy interventions. Am J Phys Med Rehabil. 2011;90(5 Suppl 1):S5-15. https://doi.org/10.1097/PHM.0b013e3182 Obe4ae.

11. Purcell A, Fleming J, Bennett S, Haines T. Development of an educational intervention for Cancer-related fatigue. Br J Occup Ther. 2010;73(7):327-33. https://doi.org/10.4276/030802210X12759925544425.

12. Henshall CL, Allin L, Aveyard H. A Systematic Review and Narrative Synthesis to Explore the Effectiveness of Exercise-Based Interventions in Improving Fatigue, Dyspnea, and Depression in Lung Cancer Survivors. Cancer Nurs. 2019;42(4):295-306.

13. Cheng $X$. Effects of lung rehabilitation therapy in improving respiratory motor ability and alleviating dyspnea in patients with lung Cancer after lobectomy: a clinical study. Altern Ther Health Med 2020 AT6400.

14. Schmitz KH, Campbell AM, Stuiver MM, Pinto BM, Schwartz AL, Morris GS, et al. Exercise is medicine in oncology: engaging clinicians to help patients move through cancer. CA Cancer J Clin. 2019;69(6):468-84. https://doi.org/1 $0.3322 /$ caac. 21579 .

15. Campbell KL, Winters-Stone KM, Wiskemann J, May AM, Schwartz AL, Courneya KS, et al. Exercise guidelines for Cancer survivors: consensus statement from international multidisciplinary roundtable. Med Sci Sports Exerc. 2019;51(11):2375-90. https://doi.org/10.1249/MSS.0000000000002116.

16. Hilfiker R, Meichtry A, Eicher M, Nilsson Balfe L, Knols RH, Verra ML, et al. Exercise and other non-pharmaceutical interventions for cancer-related fatigue in patients during or after cancer treatment: a systematic review incorporating an indirect-comparisons meta-analysis. Br J Sports Med. 2018; 52(10):651-8. https://doi.org/10.1136/bjsports-2016-096422.

17. Fernández Rodríquez EJ, Rihuete Galve MI, Cruz Hernández JJ. Impact of a comprehensive functional rehabilitation programme on the quality of life of the oncological patient with dyspnoea. Med Clin (Barc). 2020:S00257753(20)30565-0. Advance online publication.

18. Mahoney FI, Barthel DW. Functional evaluation: the Barthel Index. Md State Med J. 1965:14:61-5.

19. Mahler DA, Wells CK. Evaluation of clinical methods for rating dyspnea. Chest. 1988;93(3):580-6. https://doi.org/10.1378/chest.93.3.580.

20. Guralnik JM, Simonsick EM, Ferrucci L, Glynn RJ, Berkman LF, Blazer DG, et al. A short physical performance battery assessing lower extremity function: association with self-reported disability and prediction of mortality and nursing home admission. J Gerontol. 1994;49(2):M85-94. https://doi. org/10.1093/geronj/49.2.M85.

21. O'Driscoll BR, Howard LS, Davison AG. British Thoracic Society. BTS guideline for emergency oxygen use in adult patients. Thorax. 2008;63(Suppl 6):vi1-68.

22. Oken MM, Creech RH, Tormey DC, Horton J, Davis TE, McFadden ET, et al. Toxicity and response criteria of the eastern cooperative oncology group.
Am J Clin Oncol. 1982;5(6):649-55. https://doi.org/10.1097/00000421-198212 000-00014.

23. American College of Sports Medicine, Chodzko-Zajko WJ, Proctor DN, Fiatarone Singh MA, Minson CT, Nigg CR, et al. Exercise and physical activity for older adults. Med Sci Sports Exerc. 2009;41(7):1510-30. https://doi.org/1 0.1249/MSS.0b013e3181a0c95c.

24. Şahin S, Akel S, Zarif M. Occupational Therapy in Oncology and Palliative Care. In: Huri M, editor. In Occupational Therapy - Occupation Focused Holistic Practice in Rehabilitation. Turkey: IntechOpen; 2017. p. 207-22.

25. Gray CD, Kinnear PR. IBM SPSS statistics 19 made simple. Hove: Psychology Press; 2012. https://doi.org/10.4324/9780203723524.

\section{Publisher's Note}

Springer Nature remains neutral with regard to jurisdictional claims in published maps and institutional affiliations.

\section{Ready to submit your research? Choose BMC and benefit from:}

- fast, convenient online submission

- thorough peer review by experienced researchers in your field

- rapid publication on acceptance

- support for research data, including large and complex data types

- gold Open Access which fosters wider collaboration and increased citations

- maximum visibility for your research: over $100 \mathrm{M}$ website views per year

At BMC, research is always in progress.

Learn more biomedcentral.com/submissions 